\title{
Surfactant properties of chemically modified chitooligosaccharides and their potential application in bitumen emulsions
}

Camille Chapelle $^{1}$, Ghislain David ${ }^{1 *}$, Sylvain Caillol ${ }^{1}$, Claire Negrell ${ }^{1}$, Sylvain Catrouillet ${ }^{1}$, Myriam Desroches le Foll ${ }^{2}$, Nathalie Azéma ${ }^{3}$

${ }^{1}$ ICGM, Univ Montpellier, CNRS, ENSCM, Montpellier, France

${ }^{2}$ CST COLAS 4, Rue Jean Mermoz CS 30504 - 78771 Magny-les-Hameaux Cedex

${ }^{3}$ LMGC, IMT Mines Ales, Univ Montpellier, CNRS, Alès, France

KEYWORDS: Oligochitosan surfactant, amphiphilic structures, interfacial measurement, micellar shape, oil in water emulsions, stability

Corresponding author: Ghislain.david@enscm.fr current adress

\section{ABBREVIATIONS}

COS: chitooligosaccharides; DP: degree of polymerization; DA: acetylation degree; NHS: N-hydrosuccinimide; DCC: Dicyclohexylcarbodiimide; NMR: nuclear magnetic resonance; TOFA: tall oil fatty acid; CMC: critical micellar concentration; $A E$ : atom economy; IT: interfacial tension

\section{HIGHLIGHTS}

- Properties of chitosan-based surfactant are related to their structure

- Chitosan based surfactants present interesting interfacial properties

- Chitosan based non-ecotoxic surfactants give 55 days emulsion stabilization

\section{Abstract}

Chitooligosaccharides with degree of polymerization from 5 to 15 have been modified with different lipophilic chains, from fatty epoxide to fatty acids. Their interfacial 
properties such as critical micellar concentrations, interfacial rheology and interfacial tension were studied to establish structure-properties relationships. Their ability to selfassemble as well as their micellar shape were assessed by light scattering (dynamic and static) and transmission electron microscopic analyses. Finally, their emulsion stabilities and rheological properties were evaluated with Turbiscan and allow the conclusion that $1 \%(w / w)$ DP10 oligomers modified with Tall oil fatty acid was able to stabilize oil in water emulsions $(60 / 40 \mathrm{w} / \mathrm{w})$ for at least 55 days.

\section{Introduction}

Surfactants, occurring as simple molecules or more complex polymers, are capable of reducing surface and interfacial tensions between two immiscible phases i.e., water-oil, liquid-gas, and solid-liquid. They find applications in many aspects of our everyday life such as food, medicine, cosmetics, detergents, paints, road engineering etc [1]. In the last decades, efforts have been made to provide alternatives to surfactants derived from fossil resources. Biosurfactants [2] such as lipopeptides, sophorolipids [3], fatty acids/neutral lipids, polymeric surfactants and seed oil compounds are already available on the market [4] [5].

Cationic surfactants are defined by a positively charged hydrophilic head group [6]. They are often composed of quaternary ammonium that can be synthetic [7], fully natural such as glycine betaine [8] [9] but can also result from chemical modification of biobased moieties [10]. The toxicity of cationic amphiphilic units remains the main problem [11], hence there is a growing interest in developing ecologically friendly and biocompatible cationic surfactants using natural amino resources. Amino acids based surfactants showed great interest with reduced toxicity [12] [13] and high potential in various applications [14]. Nevertheless, amino-acids are mainly produced for animal feeding, which is why it is interesting to look for other natural amines.

Chitosan is a positively charged polysaccharide with a potential use in several areas, including applications in cosmetics, biotechnology and medicine [15] [16]. It is a 
natural nontoxic resource of potentially cationic free amines for which emulsifying properties have already been investigated [17]. It was shown that crude chitosan might be used to partially substitute industrial surfactants (up to $60 \%$ replacement) for bitumen emulsions [18]. Its suitability as Pickering emulsifier was also demonstrated several times [19] [20] [21]. In those examples, chitosan surfactants are high molar mass polysaccharides that are naturally amphiphilic. Chemical modifications may be considered to achieve even more efficient amphiphilic structures [22] [23]. Nevertheless, high molar masses chitosan shows high viscosity in solution and low reactivity. When reducing the molar mass, chitooligosaccharides (COS) can be obtained [24]. COS are easier to functionalize in order to obtain amphiphilic structures. Generally, COS are modified with fatty acids and used to form micelles for drug delivery [25] [26] or as described in our previous work, with fatty epoxide [27] and could find applications in other areas such as bitumen emulsions.

Bitumen emulsions are composed of an oily phase composed of bitumen (40 to $80 \%(\mathrm{w} / \mathrm{w}))$ and an aqueous phase $(20$ to $60 \%(\mathrm{w} / \mathrm{w}))$ that contains a surfactant (liquid or powder, between 0.1 and $2.5 \%(\mathrm{w} / \mathrm{w})$ ) [28] [29]. The bitumen is sheared through a colloid mill to disperse bitumen droplets of 1 to $20 \mu \mathrm{m}$ in the aqueous phase. Nowadays, bitumen emulsions are mainly cationic. In our previous work [27], we established the reaction conditions for the synthesis of chitosan-based surfactants from COS and fatty epoxide. We also showed their potential application in oil in water emulsion. In this study, the physicochemical properties of those sustainable chitosan-based surfactants were evaluated with surface and interfacial tension measurements combined with rheological study. Micellar shape was also investigated. Their suitability to emulsify oil has been further enhanced through deeper evaluation of destabilization processes. These potentially biobased surfactants have never been investigated beforehand and will hopefully be able to replace surfactant from petroleum in concentrated "oil in water" emulsions. 


\section{Material and method}

\subsection{Material}

Chitosan DA around 15\% CH30 (Mw $30 \mathrm{~kg} / \mathrm{mol})$ was purchased from Glentham Science and used as received; cardanol glycidyl ether was purchased from Cardolite; tall oil fatty acid (TOFA) was supplied by industrial partners (Colas); butyl glycidyl ether (C4), octyl/decyl glycidyl ether (C9), hexadecyl glycidyl ether (C16), triethylamine $\left(\mathrm{Et}_{3} \mathrm{~N}\right)$, hydroxylamine hydrochloride $\left(\mathrm{NH}_{2} \mathrm{OH}\right)$, acetic acid $(\mathrm{AcOH})$, sodium nitrite $\left(\mathrm{NaNO}_{2}\right)$, Dicyclohexylcarbodiimide (DCC), N-hydroxysuccinimide (NHS), trimethylsilyl-3-propionic2,2,3,3-D4 acid sodium salt, sodium hydroxide $(\mathrm{NaOH})$, hydrochloric acid $(\mathrm{HCl})$, and acetone (>98\%) were purchased from Sigma and used without purification; DMSO and Methanol $(\mathrm{MeOH})$ were purchased from VWR, engine oil Cirkan C 220 was purchased from Total.

\subsection{Functionalization of $\mathrm{COS}$}

\subsubsection{Synthesis of COS-epoxide}

COS of DP5 DP10 and DP15 were obtained according to precedent work [24] and functionalized with butyl glycidyl ether (C4), octyl/decyl glycidyl ether (C9), hexadecyl glycidyl ether (C16), and epoxidized cardanol according to reaction conditions established in a previous work [27]. As demonstrated beforehand, degrees of substitution (DS) were optimized to reach the higher hydrophilic lipophilic balance (HLB) while keeping water solubility (Table 1). A brown powder was obtained for each structure.

\subsubsection{Synthesis of COS-TOFA}

$0.33 \mathrm{~g}$ of TOFA was dissolved in $10 \mathrm{~mL}$ of DMSO in the presence of $0.29 \mathrm{~g}(1.2$ eq) of DCC and $0.16 \mathrm{~g}(1.2 \mathrm{eq})$ of $\mathrm{NHS}$ for 1 hour at $40^{\circ} \mathrm{C}$. The mixture was then added dropwise to $1 \mathrm{~g}$ of COS (DP5 or DP10) dissolved in 10 to $20 \mathrm{~mL}$ of DMSO. The solution was stirred for $24 \mathrm{~h}$ at $80^{\circ} \mathrm{C}$. The product was then precipitated in $200 \mathrm{~mL}$ of cold acetone, centrifuged and washed to remove impurities, residual DCC and NHS, then 
solubilized in a small volume of water and precipitated again in acetone. Finally, the product is dried under vacuum for 6 hours and a brown powder is obtained (A 1.2).

$\underline{{ }^{1} \mathrm{H} N M R}$ of DP10 + TOFA $\left(\mathrm{D}_{2} \mathrm{O}, 400 \mathrm{MHz}, 298 \mathrm{~K}, \mathrm{pH} 5.5\right): \delta$ (ppm) 8.2-8.5 (s, H aldehydes), 7.5 (d, 8Hz, 1H, Ho1 (E)-oxime) 6.8 (d, 7Hz, 1H, Ho1 (Z)-oxime), 5.35.5 (m, 6H, Hi Hh), 4.0-4.7 (m, 1H Ho2, 1H, Ho3, 1H, Ho5), 3.4-4.0 (m, 1H H2", 1H, H3; 1H, H4; 1H, H5; 2H, H6;1H, Ho4 and 2H, Ho6), $3.1(m, 1 \mathrm{H}, \mathrm{H} 2), 2.5(\mathrm{~m}, 4 \mathrm{H}, \mathrm{He}, \mathrm{Ha}), 2.0$ (s, 3H, HAc), 1.5 (m, 2H, Hb), 1.3 (m, 8H, Hc), 0.8 (m, 3H, Hd), 4.8 (s, HOD) (A.2).

\subsubsection{Characterizations}

The obtained products were characterized by ${ }^{1} \mathrm{H}$ NMR spectroscopy [27] to confirm functionalization and to calculate the substitution degree (DS) (Table 1). COS surfactants are named in function of sizes of the oligomer chain (DP5 to DP15) and of the hydrophobic chain (C4, C9, C16, Cardanol and TOFA). For example, DP15C4 is a surfactant made with an oligomer of 15 units functionalized with butylglycidyl ether C4.

HLB were calculated as follows with $M_{H}$ and $M_{L}$ the molar mass of hydrophilic and lipophilic part respectively (2). $\mathrm{M}_{\mathrm{H}}$ is actually dependent on the DP as it represents COS molar masses and $M_{L}$ represents the fatty moieties (C4, C9, C16, Cardanol and TOFA) grafted onto COS. Maximum DS and HLB are presented in Table 1.

$$
H L B=\frac{M_{H}}{M_{H}+D S \times M_{L}} \times 20
$$

Atom economy $(\mathrm{AE})$ was calculated by dividing the molar mass of the product by the sum of the molar masses of all substances produced in the stoichiometric equation [30]. For example, in the case of reactions of DP10 and DP5 with C4, C9 and C16, $\mathrm{Et}_{3} \mathrm{~N}$ was used. At the end it generates additional waste with unreacted products. Likewise, TOFA based surfactant were synthesized using DCC and NHS coupling agent, which also generates waste. AE are presented in Table 1.

$$
A E=\frac{M_{t}}{M_{t}+M_{F C}+M_{a d d}} \times 100
$$


With $M_{t}, M_{F C}, M_{a d d}$ the molar mass of the surfactant, molar mass of the unreacted fatty chains, molar masses of additives $\left(\mathrm{Et}_{3} \mathrm{~N}, \mathrm{DCC}\right.$ or $\left.\mathrm{NHS}\right)$ respectively.

\begin{tabular}{|c|c|c|c|c|}
\hline Surfactant & DS (\%) & $\begin{array}{c}\text { Number of equivalent of } \\
\text { fatty chains/ } / \mathrm{NH}_{2}{ }^{*}\end{array}$ & HLB & $A E(\%)$ \\
\hline DP15C4 & 80 & 3 & 19.2 & 53 \\
\hline DP15C9 & 47 & 3 & 19.3 & 38 \\
\hline DP15C16 & 11 & 3 & 19.7 & 25 \\
\hline DP5C4 & 100 & 1 & 17.3 & 93 \\
\hline DP5C9 & 39 & 3 & 18.3 & 39 \\
\hline DP5C16 & 25 & 3 & 18.4 & 30 \\
\hline DP5Cardanol & 12 & 3 & 19.0 & 24 \\
\hline DP5TOFA & 12 & 1 & 19.2 & 45 \\
\hline DP10C4 & 90 & 3 & 18.7 & 53 \\
\hline DP10C9 & 45 & 0.75 & 18.9 & 76 \\
\hline DP10C16 & 12 & 1 & 19.6 & 47 \\
\hline DP10Cardanol & 15 & 3 & 19.2 & 20 \\
\hline DP10TOFA & 15 & 0.3 & 19.3 & 76 \\
\hline
\end{tabular}

Table 1. Degree of substitution (DS), Hydrophilic/Lipophilic balance (HLB) and atom economy values (AE) of COS-based surfactants

*stoichiometry of fatty chain per $\mathrm{NH}_{2}$ of $\mathrm{COS}$ [27]

\subsection{Interfacial properties}

\subsection{1. $\quad$ CMCs and interfacial tensions}

The CMC and interfacial tensions at the water-oil interface of COS and modified COS solutions were determined with a Dataphysics tensiometer by using the Wilhelmy plate method. A roughened platinum plate was used. Before each measurement, the plate was rinsed with distilled water, flamed and then left to cool down. Measurements were conducted in triplicate for $\mathrm{CMC}$ determination. The results were reported as averaged \pm standard deviation.

\subsubsection{Stacking parameter}

The slope of the surface tension curve as a function of the logarithm of the concentration (A.6) allows calculating the surface concentration $(\Gamma)$ and determining the volume occupied by the hydrophilic head of the surfactant $(\mathrm{a} 0)$, according to the following equation (with $\mathrm{Na}$ the Avogadro number):

$$
\text { (1) } a 0=\frac{10^{20}}{N a \times \Gamma}
$$


The stacking parameter $(p)$, linked to the micelle shape in the solution, was calculated according to equation (18):

(2) $p=\frac{V}{\mathrm{lc} \times \mathrm{a} 0}$

With

(3) $\mathrm{V}=27,4+26,9(\mathrm{nc}-1)$

(4) $I_{c}=1,5+1,265(n c-1)$

with $\mathrm{nc}$ the number of carbon of the fatty moiety

\subsubsection{Interfacial rheology}

Interfacial rheology measurement at the water-oil interface of COS and modified COS solutions were performed on a Thermo Scientific HAAKE MARS rheometer equipped with Du Noüy ring. Experiment were performed during $30 \mathrm{~h}$ at $1 \mathrm{~Hz}$ with $10 \%$ deformation within the linear domain.

\subsection{Static and dynamic light scattering (SLS and DLS)}

Dn/dc were determined using a differential refractometer. DLS was performed on a Malvern Nanosizer ZS-apparatus equipped with a $\mathrm{He}-\mathrm{Ne}$ laser operating at a wavelength of $633 \mathrm{~nm}$. The solutions were filtered on $0.45 \mu \mathrm{m}$ nylon filters prior to use. SLS measurements were performed on a LS instrument working with concentrations C from 0.1 to and $10 \mathrm{~g} \cdot \mathrm{L}^{-1}$. Measurements were taken from 20 to $150^{\circ}$ each $20^{\circ}$ during $30 \mathrm{~s}$.

The Rayleigh ratio, $\mathrm{R}_{\theta}$, of the solution was determined as follows:

(1) $R(\theta)=\frac{I_{\text {sample }}(\theta)-I_{\text {solvent }}(\theta)}{I_{\text {toluene }}(\theta)}\left(\frac{n_{\text {solvent }}}{n_{\text {toluene }}}\right)^{2} \times R_{\text {toluene }}$

with Isample, Isolvent, Itoluene the average intensities scattered respectively by the solution, the solvent and the reference (toluene), nsolvent (1.333 for waer ) and ntoluene(1.496) the respective refractive indexes of solvent and toluene and Rtoluene $\left(1.35 \times 10^{-5} \mathrm{~cm}^{-1}\right)$ the Rayleigh ratio of toluene for a wavelength $\lambda(632.8 \mathrm{~nm})$. 
The intensity measured at each angle $\left(I_{\text {sample }}(\theta)\right)$ is then related to the concentration, the apparent molar mass $M_{a}$ of the sample and to the radius of gyration $R_{g}$ by Zimm approximation:

(2) $\frac{K C}{R(\theta)}=\left(\frac{1}{M w}+2 A_{2} C\right) \times\left(1+\frac{q^{2} \times R g^{2}}{3}\right)=\frac{1}{M a} \times\left(1+\frac{q^{2} \times R g^{2}}{3}\right)$

With $\mathrm{K}$ a constant and $\mathrm{q}$ the vertically polarized light scattering vector $\left(\mathrm{m}^{-1}\right)$

(3) $K=\frac{4 \pi^{2} n^{2} \text { solvent }}{\lambda^{4} N_{a}} \times\left(\frac{\partial n}{\partial C}\right)^{2}$

where $\mathrm{Na}$ is Avogadro's number

(4) $q=\frac{1}{\lambda} \times 4 \pi^{2} n_{\text {solvent }} \sin \left(\frac{\theta}{2}\right)$

Aggregation number was determined as followed:

(5) $N_{\text {agg }}=\frac{M w}{M_{\text {unimer }}}$

\subsection{Microscopic analysis}

Samples were observed on Transmission electron microscopy (TEM) Jeol 1200EX2 at $100 \mathrm{Kv}$. Pictures were taken by a SIS Olympus Quemesa equiped with a digital camera with a 11 Mpixel CCD sensor. The point-to-point resolution of TEM was $0.4 \mathrm{~nm}$ and its resolution on gratings was $0.2 \mathrm{~nm}$.

\subsection{Emulsion}

\subsubsection{Preparation}

Motor oil was used to simulate bitumen. Cirkan 220 motor oil shows indeed the same viscosity at $40^{\circ} \mathrm{C}$ than bitumen at $150^{\circ} \mathrm{C}$. Oil-in-water emulsions were produced as follows: surfactant was dissolved in $0.2 \% \mathrm{w} \mathrm{HCl}$ aqueous solution $(40 \%(\mathrm{w} / \mathrm{w})$ of the total emulsion). Both oil and water phases were heated at $40^{\circ} \mathrm{C}$. Oil $(60 \%(\mathrm{w} / \mathrm{w})$ of the total emulsion) was added to the aqueous phase and blended for 5 min with an Ultraturax at $19000 \mathrm{rpm}$.

\subsubsection{Stability}

Stability of motor oil emulsions was evaluated by Turbiscan Lab [31] for a period of 55 days maximum. Backscattering (BS) and transmission (T) are directly linked to the 
mean particle diameter $d$ and $\phi$ the oil volume fraction $(v)$ of the sample with $r_{i}$ the internal radius of the measurement cell $(\mu \mathrm{m}), \lambda^{*}$ the photon transport length $(\mu \mathrm{m}), \mathrm{g}$ the scattering efficiency factor and Qs the asymmetry factor:

$$
\begin{aligned}
& \mathrm{BS}=\left[\frac{1}{\lambda^{*}}\right]^{\frac{1}{2}} \\
& \mathrm{~T}=e^{\frac{-r_{i}}{\lambda}} \\
& \lambda^{*}=\frac{2 d}{3 \phi(1-g) Q s} \\
& \lambda=\lambda^{*}(1-g)
\end{aligned}
$$

Emulsions were directly analyzed and also diluted $1 / 3$ in water to speed up the destabilization process. Clarification kinetics on the first 20 minutes, average diameter and relative height of each phases were quantified for each surfactant. A destabilization index at 7 days (D7days) was calculated according to the lower clarification and oil phase formation on the top of the emulsion as follows:

(9) D7days $(\%)=100 \times \frac{h D+\text { hoil }}{h_{E m}}$

With $h_{D}, h_{o i l}, h_{E m}$ the height of the dispersion phase, oily phase after 7 days and height of emulsion at t0 respectively.

Measurements were conducted in triplicate for one sample to evaluate standard deviation on destabilization kinetics, 7 days destabilization (D7days) and average diameter.

\subsubsection{Bulk rheology}

Bulk rheology measurement were performed on a Thermo Scientific HAAKE MARS rheometer equipped with a coaxial cylinder geometry. Experiment were performed at $1 \mathrm{~Hz}$ with $10 \%$ deformation within the linear domain.

\subsection{Surfactant toxicity}


Toxicity and ecotoxicity of surfactants of interest were evaluated according to acute immobilization tests on Daphnia magna (NF EN ISO 6341, 2012) performed by Eurofins. This test aims at finding the concentration immobilizing $50 \%$ of the daphnia population after $24 \mathrm{~h}$ and $48 \mathrm{~h}$ (CE50). A solution of $\mathrm{K}_{2} \mathrm{Cr}_{2} \mathrm{O}_{7} \mathrm{CE} 50-24 \mathrm{~h}=0,94 \mathrm{mg} / \mathrm{L}$ (this value corresponds to standard between $0.60 \mathrm{mg} / \mathrm{L}$ and $2,1 \mathrm{mg} / \mathrm{L}$ according to NF EN ISO 6341: 2012) is used as a reference substance. Samples were prepared by dissolving surfactants in an ultra-pure water solution of anhydrous sodium hydrogen carbonate $\mathrm{NaHCO}_{3}(0.200 \mathrm{~g} / \mathrm{L})$, calcium chloride $\mathrm{CaCl}_{2}(0.260 \mathrm{~g} / \mathrm{L})$, magnesium chloride $\mathrm{MgCl}_{2}, 6$ $\mathrm{H}_{2} \mathrm{O}(0.148 \mathrm{~g} / \mathrm{L})$, anhydrous potassium sulfate $\mathrm{K}_{2} \mathrm{SO}_{4}(0.026 \mathrm{~g} / \mathrm{L}) .100 \mathrm{mg} / \mathrm{L}$ solutions were prepared and diluted as followed: $35 \%, 10 \%, 3.5 \%$, and $1 \%(\mathrm{w} / \mathrm{w})$. Tests were performed at $20^{\circ} \mathrm{C}$.

\section{Result and discussion}

COS based surfactants were obtained by grafting butylglycidyl (C4), octyl/decyl (C9), hexadecyl (C16) ethers, epoxidized cardanol and C18 fatty acid (TOFA) on DP5, DP10 and DP15 oligomers (Figure 1). In the following study, interfacial properties, micellar shape of each surfactant and emulsion stability were studied in order to establish structure property relationships and evaluate their efficacy in oil in water emulsion application.

\subsection{CMC Measurement}

The critical micellar concentration $(\mathrm{CMC})$ is defined at a given temperature as the concentration of surfactant from which it spontaneously self-assembles to form micelles. It is directly related to the surface tension (ST) that represents the tension existing at the interface between air and the surfactant. Cationic surfactants usually possess higher CMCs than nonionic ones. In fact, surfactants used in bitumen emulsions generally show

CMCs between 0.1 and $1 \mathrm{~g} \cdot \mathrm{L}^{-1}$. CMCs measurement of COS-based surfactant is therefore important in order to assess their suitability for the application. 
First, it was observed that unmodified COSs lower the water surface tension from $71 \mathrm{mN} \cdot \mathrm{m}^{-1}$ to 47 to $55 \mathrm{mN} \cdot \mathrm{m}^{-1}$ depending on the DP. Their modification with $\mathrm{C} 4$ does not led to significant reduction of this tension, nonetheless, the grafting with fatty moieties (C9, C16, TOFA and Cardanol) allows reducing even further this tension between 41 and $32 \mathrm{mN} \cdot \mathrm{m}^{-1}$ (Table 2). As expected, COS functionalization provides a significant surfactant aspect to COS. CMC of each surfactant was measured at $25^{\circ} \mathrm{C}$ in aqueous solution at $\mathrm{pH} 2$ (Table 2). CMCs were lower than $1 \mathrm{~g} \cdot \mathrm{L}^{-1}$ (Table 2). This was not the case for crude COS and COS modified with C4 fatty epoxide for which no CMC could be determined. $\mathrm{C} 20$, i.e. the concentration for which the surface tension is reduced by $20 \mathrm{mN} \cdot \mathrm{m}^{-1}$, was also calculated for each surfactant. It was shown that it is quite high for DP10 C16, indicating lower surfactant properties. In the same way than CMCs, C20 of both unmodified COS and C4-modifed COS surfactants could not be determined, being higher than 2 g. $\mathrm{L}^{-1}$.

\begin{tabular}{|c|c|c|c|c|c|c|}
\hline Surfactant & $\begin{array}{l}\text { CMC } \\
\left(\text { g. } L^{-1}\right)\end{array}$ & C20 (g. $\left.\mathrm{L}^{-1}\right)$ & $\begin{array}{l}\text { Surface } \\
\text { tension } \\
\mathrm{C}=2 \mathrm{~g} \cdot \mathrm{L}^{-1} \\
\left(\mathrm{mN} \cdot \mathrm{m}^{-1}\right)\end{array}$ & $\begin{array}{c}\text { Stacking } \\
\text { parameter } \mathrm{p}\end{array}$ & $\begin{array}{l}\text { Fatty chain } \\
\text { per } \\
\text { surfactant }\end{array}$ & $\begin{array}{l}\text { Free amine } \\
\text { per } \\
\text { surfactant }\end{array}$ \\
\hline DP15 & $>2$ & $1.89 \pm 0.02$ & $47.5 \pm 1.0$ & - & 0 & 12.5 \\
\hline DP15C4 & $>2$ & $1.27 \pm 0.02$ & $49.1 \pm 1.2$ & - & 10 & 2 \\
\hline DP15C9 & $0.576 \pm 0.008$ & $0.21 \pm 0.02$ & $41.4 \pm 0.3$ & 0.14 & 6 & 6 \\
\hline DP15C16 & $0.587 \pm 0.005$ & $0.08 \pm 0.03$ & $41.8 \pm 0.6$ & 0.13 & 2.5 & 10 \\
\hline DP5 & $>2$ & $1.44 \pm 0.04$ & $49.6 \pm 2.8$ & - & 0 & 3 \\
\hline DP5C4 & $>2$ & $>2$ & $53.0 \pm 0.3$ & - & 3 & 0 \\
\hline DP5C9 & $0.698 \pm 0.027$ & $0.04 \pm 0.01$ & $36.5 \pm 0.3$ & 0.13 & 1 & 2 \\
\hline DP5C16 & $0.792 \pm 0.098$ & $0.07 \pm 0.08$ & $40.4 \pm 0.7$ & 0.14 & 0.7 & 2.3 \\
\hline DP5Cardanol & $0.754 \pm 0.002$ & $0.41 \pm 0.01$ & $40.2 \pm 0.3$ & 0.19 & 0.3 & 2.7 \\
\hline DP5TOFA & $0.712 \pm 0.008$ & $<0,01$ & $33.3 \pm 0.2$ & 0.09 & 0.3 & 2.7 \\
\hline DP10 & $>2$ & $>2$ & & - & 0 & 7.5 \\
\hline DP10C4 & $>2$ & $>2$ & $52.8 \pm 1.2$ & - & 6.8 & 0.7 \\
\hline DP10C9 & $0.229 \pm 0.011$ & $<0,01$ & $31.8 \pm 0.6$ & 0.22 & 3.3 & 4.2 \\
\hline DP10C16 & $0.775 \pm 0.009$ & $1.03 \pm 0.04$ & $54.4 \pm 0.4$ & 0.19 & 0.9 & 6.6 \\
\hline DP10Cardanol & $0.401 \pm 0.011$ & $0.07 \pm 0.04$ & $41.0 \pm 0.9$ & 0.07 & 1.1 & 6.4 \\
\hline DP10TOFA & $0.558 \pm 0.009$ & $<0,01$ & $33.4 \pm 0.3$ & 0.20 & 1.1 & 6.4 \\
\hline
\end{tabular}

Table 2. CMC, C20 and stacking parameters values of COS-based surfactants

It is possible to assess the performance and efficiency of one surfactant over another by looking at the CMC values and its associated surface tension [32]. 
Performance involves the adsorption capacity at interfaces and is related to surface tension, while efficiency defines the self-aggregation capacity of surfactants related to CMC. For example, when the surface tension after the CMC of a surfactant $A$ is lower than that of a surfactant $B$, i.e. $\gamma_{\triangle M C ~} A<\gamma_{C M C B}$, the surfactant $A$ will be described as more efficient than $B$. When $C M C$ value of a surfactant $C$ is lower than that of a compound $D$, i.e. $\mathrm{CMC}_{\mathrm{C}}<\mathrm{CMC}_{\mathrm{D}}$, the surfactant $\mathrm{C}$ will be described as more efficient than $\mathrm{D}$. In this study, we were able to classify the surfactants as follows according to their efficiency: DP10C9 > DP10Cardanol > DP10TOFA > DP15C16 > DP15C9 > DP5C9; DP5Cardanol; DP5TOFA > DP5C16 > DP10C16 and for performance: DP5C9; DP5TOFA; DP10C9; DP10TOFA > DP5C16 > DP5Cardanol; DP10Cardanol >> DP10C16 > DP15C9;

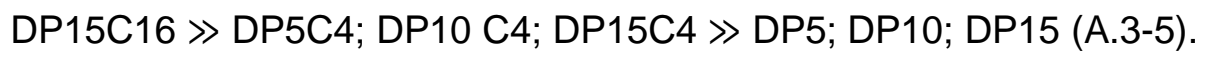

First, we can observe a relationship between CMCs and the number of fatty carbons for the same hydrophilic chain length. For example, with DP10, the ranking is DP10C9>DP10Cardanol>DP10TOFA>DP10C16 which is in agreement with their respective fatty carbon numbers and grafting efficiency i.e. 30 (3.3 C9 chains), 23 (1.1 Cardanol), 20 (1.1 TOFA), 14 (0.9 C16). In addition, increment of CMCs was linked to insaturation number on the fatty chains, which explains the higher values for TOFA and cardanol surfactants [33]. With DP5 surfactants, poor grafting efficiency is reached. Their CMC values are close to each other but are higher than that of DP10s due to a larger amount of unmodified COS in the mixture. DP5 to DP15 COS modified with C4 were out of ranking with higher DS but no CMC could be measured. This means that the chain length of one graft is more important than the total number of carbons at some point and 4 carbons are not effective enough to afford surfactant properties.

The performance can also be related to the general structure of surfactants. Indeed, the performance depends mostly upon the size of the COS. Oligomers are less efficient when the hydrophilic chain is longer, i.e; DP15《DP10;DP5. With DP10 and DP5, other factors such as DS and hydrophobic chain length have to be taken into 
account. Indeed, higher surface tensions are obtained for COS functionalized with C4, i.e. values rather close to COS itself. DP10C16 shows quite high surface tension similar to that of $\mathrm{C} 4$ based surfactants. Finally, it was shown that it is necessary to graft fatty chains with at least 9 carbons onto $\operatorname{COS}$ or 5 or 10 units to obtain interesting properties. Indeed, oligomers of DP5 and DP10 functionalized with C9, TOFA and Cardanol showed both competitive $\mathrm{C} 20$ and surface tensions.Stacking parameters were calculated according to equations $(1,2,3$ and 4$)$ regarding to the length of the hydrophobic part (A.6). Stacking parameters of surfactants were less than 0.33 , enhancing the formation of direct and spherical micelles.

\subsection{Interfacial tension}

Interfacial tensions between motor oil (model for bitumen) and surfactant solutions were determined according to the Wilhelmy method [34]. Interfacial tension expresses the force per unit area that pulls molecules at the interface inward the liquid. In general, the efficiency of an emulsifier can be evaluated by its capacity to reduce the interfacial tension between oil and water: the lower is the interfacial tension, the higher is the wateroil affinity and the better is the surfactant stabilization of the oil-in-water emulsion. All measurements were carried out at $\mathrm{pH} 2$, which is the $\mathrm{pH}$ of the aqueous phases in bitumen emulsions. Figure 2 shows the interfacial tension values between oil and water, crude and modified COS solutions at the water/oil interface.

Initially, it was shown that unmodified COS (i.e. DP5, DP10 and DP15) allow the interfacial tension (IT) to be reduced almost by half, i.e. from $44 \mathrm{mN} / \mathrm{m}$ to 26,24 and $26 \mathrm{mN} / \mathrm{m}$, respectively (Figure 2 dark blue bars). It was noted that despite significant DS (Table 1), the addition of C4 had almost no impact on interfacial tension compared to unmodified COS. Thus, both ITs and CMCs values allowed concluding that C4 group is not hydrophobic enough to provide good surfactant properties to COS, whatever the DP value. Nevertheless, when increasing the hydrophobic chain (i.e. C9, C16, TOFA and Cardanol), IT values were strongly reduced. This decrease was due to the fatty chains 
addition that enhanced the amphiphilicity, thereby remarkably improving the surface activity of COS. It is however to be noted that this decrease was less pronounced with DP15, probably due to a higher number of free amines, leading to a stronger hydrophilic part (Table 2). High IT value for DP10C16 can be explained by a low rate of grafting, i.e. DS of $12 \%$ which reflects 0.9 C16 group per COS chain. In addition, it is a shorter chain length compared to DP10TOFA and DP10Cardanol that are made of 1.1 chains of 18 and 21 carbons respectively. However, some of the surfactants allowed decreasing TI value by almost $80 \%$. According to $\mathrm{TI}$ values, COS-based surfactants showing the best performances can be ranked as follows: DP10C9 > DP5C16> DP5C9 $\geq$ DP10TOFA> DP5TOFA > DP5Cardanol> DP10Cardanol.

In summary, the TI depends on different structural factors such as the DP, the size and structure of the fatty chain, the DS and the number of free amines (see Table 2). Hydrophilic chain length is playing an important role in the surfactant properties. Despite high grafting efficiency, DP15 led to lower interfacial properties than DP10 and DP5. For example, DP15C9 led to higher IT, CMC and C20 values despite twice more fatty chains than DP10C9. In addition, the influence of the hydrophobic chain length was also crucial. Indeed, C4 based surfactants showed poor interfacial properties despite high grafting efficiency. For example, DP10C4 has the same content of fatty carbons, distributed differently, than DP10C9. Nevertheless, DP10C9's IT was more than twice lower than DP10C4'. In addition, with close DSs i.e. 0.9 and 1.1, and equal DPs (DP10C16, DP10Cardanol and DP10TOFA) ITs values were related to the hydrophobic chain length and structure. Indeed, C16 comes first with high IT value. It is followed by cardanol with an aromatic ring and a 15 carbons chain and finally TOFA with 18 carbons unsaturated chains. In fact, TOFA-based surfactant showed better properties than cardanol-based surfactant probably due to the steric hindrance and stiffness induced by the aromatic ring.

These results combined with previous ones led to a first selection for the rest of the study. C4 and DP15 based surfactants were not considered due to poor surface and 
interfacial properties. DP10C16 and DP5C16 were not used due to less interesting properties and low reaction yield.

\subsection{Static and dynamic light scattering (SLS and DLS)}

When dispersed in water above CMCs, surfactants self-assemble into micelles. Their sizes and shapes can be checked by light scattering analysis. SLS allows to check the shape and calculate the molar masses of the aggregates leading to the number of surfactant per micelles. Results are presented in table 3.

\begin{tabular}{lccccccc}
\hline \multicolumn{1}{c}{ Surfactant } & $\mathrm{Rh}(\mathrm{nm})$ & $\mathrm{dn} / \mathrm{dc}$ & $\begin{array}{c}\mathrm{M}_{\text {unimer }} \\
(\mathrm{g} / \mathrm{mol})\end{array}$ & $\begin{array}{c}\mathrm{Mn} \\
(\mathrm{g} / \mathrm{mol})\end{array}$ & Nagg & $\mathrm{Rg}(\mathrm{nm})$ & $\mathrm{Rg} / \mathrm{Rh}$ \\
\hline DP5Cardanol & 110 & 0.1528 & 982 & 25,500 & 27 & 85 & 0.8 \\
DP5TOFA & 130 & 0.1424 & 943 & 44,400 & 49 & 100 & 0.8 \\
DP5C9 & 150 & 0.1795 & 1,077 & 23,000 & 22 & 170 & 1.1 \\
DP10Cardanol & 110 & 0.1920 & 2,028 & 57,000 & 28 & 77 & 0.8 \\
DP10TOFA & 45 & 0.1146 & 1,955 & 33,000 & 17 & 65 & 1.4 \\
DP10C9 & 60 & 0.1652 & 2,275 & 51,300 & 22 & 75 & 1.3 \\
\hline
\end{tabular}

Table 3. Molar masses, aggregation number and gyration radius of COS-based surfactants

At first, the hydrodynamic radius $\mathrm{Rh}$ was measured by DLS. It was shown that sizes were quite homogeneous between 110 and $150 \mathrm{~nm}$ except for DP10TOFA and DP10C9 with lower values, 45 and $60 \mathrm{~nm}$ respectively. In the literature, chitosan based micelles are usually described to be between 30 and $150 \mathrm{~nm}$ which is in accordance with our results [37] [38]. The molar masses of the micelles were obtained thanks to Zimm approximation (equations $1-5$ and A.9-14). This led to the aggregation number Nagg, which represents the number of surfactant molecules inside a micelle. No significant difference was observed according to DP or lipophilic chain length with values between 17 and 28. Higher Nagg was obtained for DP5TOFA which demonstrated nice selfassembly properties. Both DP10TOFA and DP10 C9 with lower hydrodynamic diameters showed the same order of magnitude in molar masses and Nagg than the other surfactants. In addition, the radius of gyration $\mathrm{Rg}$ was determined by SLS analysis. $\mathrm{Rg} / \mathrm{Rh}$ Ratios $\leq 0.8$ mean that micelles are spherical. Micelles were observed under TEM to check their spherical shape (Figure 3). In our case, the higher $\mathrm{Rg} / \mathrm{Rh}$ values of $1.1,1.4$ and 1.25 for DP5C9, DP10TOFA and DP10C9 respectively were attributed to higher 
dispersity in the size distribution and possible aggregation of the micelles which was illustrated by TEM analysis (Figure 3 b)). Additionaly, no signal was obtained when depolarized dynamic light scattering was performed on the samples proving the absence of elongated micelles.

\subsection{Emulsion stability and rheological properties}

4.4.1. Stability over 7 days

Surfactants were used to emulsify a motor oil in water $(60 / 40 \mathrm{w} / \mathrm{w})$ as a model for bitumen emulsions. The stability of emulsions was evaluated with Turbiscan to highlight the destabilization processes and kinetics in order to discriminate the efficiency of COSbased surfactants. In bitumen emulsions, surfactant usually represent $0.2 \%(\mathrm{w} / \mathrm{w}$ of emulsions). Commercial surfactants are usually unimers constituted of one hydrophobic tail grafted onto one polar head. In our case, different grafting efficiencies, hydrophobic and hydrophilic chain lengths have to be compared. This is why COS-based surfactants were used in equimolar amount (5.48E-04 mol) with their respective mass in the emulsion, calculated according to their molar masses (Table 4).

Emulsions were at first threefold diluted to accelerate destabilization processes. Figure 5 shows the profile evolution of oil-in-water emulsion obtained with DP10 TOFA during 7 days (Figure 4 a)). Different phenomena could be identified from this evolution profile and linked to visual observation of the column (Figure 4 b)). At t0, emulsion represented most of the samples and foams was observed at the surface, due to the mixing process. No transmission was observed and high stable backscattering values (around $70 \%$ ) were observed meaning that emulsion was homogeneous in concentration and size drops.

During the first 20 minutes, a lower clarification (LC) was underlined by backscattering profiles evolution ( 0 to $20 \mathrm{~mm}$ Figure 4 a) ) but no transmission was observed. Only after several days transmission could be observed on the first millimeters (0-10 mm). Nevertheless, clear water or surfactant solution was not reached after 7 days 
as the maximum transmission was $70 \%$, which is far from the transmission of the surfactant in solution, i.e. between 80 and $90 \%$ (Table A.1).

The second phenomenon was the disappearance of the foam that can be followed at the top of the column by emulsion's front motion.

After 7 days, two distinct phases standed out (Figure 4 b)). The lower one was an emulsion with a vertical increasing concentration in oil particles $(0-20 \mathrm{~mm})$, called dispersion in the rest of the study, and a supernatant emulsion phase (20-32 mm). For higher destabilization, this supernatant can be divided into two parts: a first layer (20$26 \mathrm{~mm}$ ) with a very high concentration of oil particles (BS increases as a consequence of oil transfer from dispersion phase to supernatant) and a second layer mainly made of oil (26-32 $\mathrm{mm})$ due to a higher coalescence of oil particles.

Emulsions made with DP5TOFA, DP10Cardanol and DP5Cardanol showed the same profiles than that of DP10TOFA (A.15-17) with more or less 20 millimeters of dispersion after 7 days (Figure 5 c)). However, DP5C9 and DP10C9 showed higher destabilization with an additional upper oily phase (A.13-14).

\begin{tabular}{lcccc}
\hline Surfactant & $\begin{array}{c}\text { Mass of TA } \\
\text { (\% emulsion) }\end{array}$ & $\mathrm{d}_{\text {initial }}( \pm 0.7 \mu \mathrm{m})$ & $\begin{array}{c}\text { Clarification } \\
\text { kinetics }( \pm 1.6 \\
\mathrm{mm} / \mathrm{h})\end{array}$ & D7 days (\%) \\
\hline DP10 TOFA & 1.08 & 9.8 & 20.9 & 64 \\
DP10 Cardanol & 1.13 & 13.1 & 35.3 & 66 \\
DP5 Cardanol & 2.47 & 11.5 & 50.4 & 68 \\
DP5 TOFA & 1.75 & 10.3 & 27.0 & 68 \\
DP10 C9 & 1.26 & 11.8 & 30.6 & 80 \\
DP5 C9 & 0.58 & 25.4 & 124.6 & 90 \\
\hline
\end{tabular}

Table 4. Mean diameters, clarification kinetics and destabilization index at 7 days of emulsions stabilized with COS-based surfactants (determined with Turbiscan Lab).

The destabilization index at 7 days (D7days) was calculated according to the lower clarification and oil phase formation on the top of the emulsion. As a result, DP10C9 and DP5C9 showed higher D7days than oligomers modified with TOFA or Cardanol, i.e. $80-90 \%$ as opposed to $64-68 \%$ respectively. 
Clarification kinetics can be determined from the peak width evolution versus time (A.18) curve (slope from the origin during the first $20 \mathrm{~min}$ ). This peak width was measured in delta mode (A.15) corresponding to the subtraction of the first profile from following profiles. Clarification kinetics for each surfactant are given in Table 4. Mean diameters can also be calculated from each of the profiles. It was shown that mean diameter of the particles of oil is directly linked to the destabilization kinetics. In fact, it is well known that the granulometric distribution plays an important role in emulsion stability. In the case of bitumen emulsions, particles sizes close to $10 \mu \mathrm{m}$ are usually reached [39] [40]. This relationship was confirmed with COS-based surfactants with destabilization kinetics steadily increase with diameter. According to kinetics of destabilization, efficiency of COS-based surfactants followed this order: DP10TOFA> DP5TOFA> DP10C9> DP10Cardanol> DP5Cardanol 〉DP5C9. DP5C9 destabilization was fast, i.e. six time faster than DP10TOFA. In addition as mentioned beforehand, an upper phase highly concentrated in oil appeared. DP10 C9 destabilization kinetics was correct but the upper oily phase formed, highlighting an additional destabilization process (80\%). DP10TOFA and DP10 Cardanol showed the lower destabilization ratio with 64 and $66 \%$ respectively followed by DP5TOFA and DP5Cardanol with 68\%. Finally, despite better interfacial properties, DP5C9 and DP10C9 did not seem to perform well as COS-based surfactant for oil-in-water emulsion stabilization. In fact, DP10C9 bears approximatively 27 fatty carbons divided on three C9 grafts against one 18 carbons chain for DP10TOFA (Table 2). In other words, the chain length but not the number of fatty carbons seems to govern the emulsion stability. Higher surfactant concentration and/or lower oil concentration could be considered to increase the stability using C9 COS-based surfactants. Cardanol COS-based surfactant showed little less interesting properties than TOFA ones despite the aromatic cycle but are competitive. It might be explained by the fact that the $\mathrm{C} 15$ fatty chain governs the interfacial properties of cardanol COS-based surfactants. All this data were gathered on radar diagram for more clarity (Figure 5) with the best values on the edge of the web. This diagram confirmed that DP10 surfactants show better properties 
that their DP5 analogues and that C9 surfactants are not homogeneous in term of properties.

\subsubsection{Interfacial and bulk rheology}

It was shown beforehand that DP5 surfactant showed less interesting properties than their DP10 analogues. This is why, going further, the characterizations were focused on DP10 surfactants. It was shown previously that DP10 Cardanol and DP10TOFA showed the smaller destabilization (D7days) values. Despite high D7days, DP10C9 showed interesting interfacial and surface tensions properties. Consequently, characterizations of DP10 surfactants were complemented by interfacial rheology to get more insight on the results previously obtained. Indeed, interfacial rheology depends on interfacial adsorption and intermolecular interactions and can testify of the capacity of the surfactant to stabilize the emulsion. Adsorption, aging and structural re-organization of the surfactants at the oil water interface was evaluated by interfacial rheology through the Du Noüy ring method [35].

At first, interfacial rheology measurements were performed at $\mathrm{pH} 2$ in water solutions and DP10 solutions to have a reference (A.19). In both cases, the elastic moduli was not stable over time which demonstrates a liquid like behavior. On the contrary, it was shown that the interfacial storage moduli (Gi') of the interface formed between DP10TOFA or DP10Cardanol solutions $\left(2 \mathrm{~g} \cdot \mathrm{L}^{-1}\right.$ ) and motor oil increases (from $10^{-5}$ to $10^{-4}$ $\mathrm{mN} \cdot \mathrm{m}^{-1}$ ) over several hours to reach a plateau after $24 \mathrm{~h}$ (Figure 6). This phenomenon was already described before in the literature with chitosan-silica based Pickering emulsions [21] and corn fiber gum emulsifier [36]. This indicates the formation of a strong interfacial layer between oil and surfactant solutions. Indeed, the adsorption of the surfactant at the oil-water interface leads to significant increase of the elastic modulus. With DP10C9, the interfacial elastic modulus was not stable over time. In fact, at first it shows promising properties with an increase of the Gi'. Over time, no plateau was reached and the modulus started to decrease instead. This means that the interfacial film 
is not strong enough and breaks over time. In addition, the viscous modulus was measured (Figure $3 \mathrm{~b}$ ) for all surfactants. DP10Cardanol and DP10TOFA showed lower Gi" compared to DP10C9 (Figure 6 b)) and DP10 solution and water itself (A.20). This is in accordance with the previous observation. Indeed, the viscous modulus is higher because of the interfacial gel weakness testifying a liquid like behavior. At the end, DP10Cardanol showed little higher interfacial storage modulus than that of DP10TOFA solutions, i.e. $1.5 \cdot 10^{-4}$ and $1 \cdot 1 \cdot 10^{-4} \mathrm{mN} \cdot \mathrm{m}^{-1}$ respectively which can be translated as a stronger affinity between oil and water with DP10Cardanol.

In the end, DP10TOFA and DP10Cardanol were confirmed to be the best candidates for emulsion stabilization. Bulk rheology of emulsion, using 1 and $1.1 \% \mathrm{w} / \mathrm{w}$ of DP10TOFA and DP10Cardanol respectively, were therefore performed to gain more insights on the emulsion maturation in time through viscosity measurement.

The aim of this study was to evaluate the evolution of the rheological properties of the emulsions over 7 days. First, it was shown that both surfactants led to emulsions with close viscosity at low shear rate, 0.1 and $0.4 \mathrm{~Pa} . \mathrm{s}$, respectively. Then, it was shown that viscosity of emulsions increased over time. Indeed, at low share rate, viscosity values after two days were 10.5 and 7.5 Pa.s respectively. No significant evolution was observed between 2 and 7 days as emulsions were quite stable. This increase of the viscosity over 2 days can be linked to the interfacial rheology measured beforehand. Indeed, it was shown above that the organization of surfactants at the oil-water interface is a long process aging for at least $24 \mathrm{~h}$. This can explain the viscosity changes over time during the first two days as particles may re-organize to reach a most stable state. This was also confirmed by frequency sweep measurement (Figure $7 \mathrm{c}$ )). Indeed, at t0, the viscous modulus (G') is higher than the elastic modulus ( $\left.G^{\prime}\right)$ inducing a liquid like behavior. After 2 days, G' value increased quite importantly and gets higher than G", droplets surrounded by surfactant leading to emulsion thickening. An intersection point can be observed at high frequency witnessing gel breaking. The same behavior was 
observed for both surfactants but DP10TOFA emulsion showed higher G' and G" values at the end. After 7 days, no further evolution of both elastic and viscous modulus was observed. In addition, it was shown that the aged emulsions were shear-thinning. Indeed, the viscosity is getting lower when increasing the shear rate. Interestingly, when decreasing the shear rate (half-filled buttons) afterwards, the viscosity of the non-aged emulsion (at t0) was reached again (Figure 7 a)). This means that the thickening phenomenon of the emulsion is reversible upon shearing. This is an important property for emulsion dispersion and application. Nonetheless, after 5 days, the viscosity is back to higher values which means that after a certain time, particles tend to aggregate again and lead to emulsion thickening.

Increase of viscosity over time is a conventional phenomenon in bitumen emulsions. To make the emulsion fluid again, the emulsion can be heated. In our case, we heated both emulsions at $40^{\circ} \mathrm{C}$. It was shown for DP10TOFA emulsions that the heating led to lower viscosity at low shear rate (Figure 7 b)). The fluidity was also confirmed by frequency sweep. Indeed, lower modulus was obtained and a liquid behavior was observed with G">G' (Figure $7 \mathrm{~d}$ )). This means that an increase of the temperature allows reaching lower viscosity without mixing. When mixing, even lower viscosity can be obtained (increasing share rate Figure 7 b)). In the case of DP10Cardanol emulsion, the viscosity is higher with the temperature (A.21.b). In addition, the decreasing share rate showed the same behavior that increasing share rate which means that the interactions were formed again in a short period of time. The gel like behavior is stronger with higher G' values and intersection point at higher frequency. Those results assess the thickening effect of DP10Cardanol surfactant with temperature.

\subsubsection{Stability over 55 days}

At the end, both DP10TOFA and DP10Cardanol showed interesting properties in emulsion. DP10Cardanol show thickening properties that was not sought in this case and 
showed Atom Economy (AE) value more than twice lower than DP10TOFA one (Table 1). Consequently, DP10TOFA at $1 \% \mathrm{w} / \mathrm{w}$ was chosen for the last part of the study.

It was used to emulsify motor oil to evaluate the stability over time (Figure 8). It was shown that lower clarification happened during the first 48 hours to reach $22 \%$ of destabilization. In this case, $85 \%$ transmission was reached for the entire height of the clarification phase. This means that the lower phase is constituted of a DP10TOFA solution in water between 0.1 and $2 \mathrm{~g} \cdot \mathrm{L}^{-1}$ (Table A.1). After 2 days, no significant evolution was observed. This evolution is interesting as it can be correlated with interfacial rheology measurement. Indeed, it was shown above that the organization of surfactants at the oil-water interface is a long process aging for at least $24 \mathrm{~h}$. At the same time, an increase of the mean diameter was shown, from 6.9 to $8.4 \mu \mathrm{m}$ in the first two days. This increment can be linked with the previously measured viscosity increase over two days. Indeed, granulometry has a huge impact on emulsions viscosity and coalescence leads to higher viscosities. Measurements were stopped after 55 days and the emulsion was still stable. In addition, the emulsion was still direct and diameters were stable between 2 and 55 days.

\subsection{Surfactant toxicity}

Ecotoxicity of surfactants i.e. DP5 and 10 functionalized with C9, TOFA and cardanol, was evaluated, according to acute immobilization tests on Daphnia magna. Each experiment was reproduced 4 times on a population of five daphnia. It was shown

that after $48 \mathrm{~h}$, the daphnia used for the test were still mobile for all the COS-based surfactants at concentration of $100 \mathrm{mg} \cdot \mathrm{L}^{-1}$ and below (Table A.2). Those results assessed that COS-based surfactants are not acute toxic/ecotoxic and their CE50-24h and CE50$48 \mathrm{~h}$ are above $100 \mathrm{mg} \cdot \mathrm{L}^{-1}$. In fact they show very interesting properties as they are far less ecotoxic than some other bio based cationic surfactant from lysine [41].

\section{Conclusion}


In this work, the interfacial properties of chitosan-based surfactants were evaluated. COS of DP5 to DP15 modified with fatty epoxides (C4, C9, C16 and cardanol) or fatty acid (TOFA C18) were investigated as potential surfactants to emulsify bitumen. COS-based surfactants appeared to be nontoxic and potentially $100 \%$ biobased. It was shown that surfactants properties are dependent on both hydrophilic and hydrophobic chain lengths and grafting efficiency but mainly dependent on the fatty chain added. As a matter of fact, surfactants functionalized with C4 showed poor interfacial properties compared to longer chains. In addition, longer hydrophilic moieties such as DP15 gave lower values than its shorter analogs. Consequently, structure / property relationships were established for the entire range of synthetic surfactants. Finally, emulsifying properties were evaluated by measuring destabilization kinetics. It was proved that particles diameters were linked to the emulsion stability as follow: the smaller is the diameter, the better is the stability. The destabilization process was observed and studied for DP5 and DP10 surfactants grafted with C9, TOFA and Cardanol. At the end, DP10TOFA seemed to be the best candidate with interesting interfacial properties and ability to stabilize $60 / 40$ oil in water emulsion for 55 days.

\section{AUTHOR CONTRIBUTION:}

The manuscript was written through contributions of all authors. The experiment were performed by C.Chapelle. S.Catrouillet helped with light scattering methods. N.Azema helped with Turbiscan. C.Chapelle wrote the manuscript with in-depth editing from G.David, C.Negrell, S.Caillol, M. Desroches and contributions from all authors. All authors have given approval to the final version of the manuscript.

COMPETING INTEREST:

Authors declare non competing financial interest.

\section{FUNDING}

This work was funded by a private funding and a CIFRE grand from the ANRT. 
The author thanks Mimoune Abadassi for his help with turbiscan analysis. 
[1] N. Kumar, R. Tyagi, Industrial Applications of Dimeric Surfactants: A Review, J. Dispers. Sci. Technol. 35 (2014) 205-214.

https://doi.org/10.1080/01932691.2013.780243.

[2] D.G. Hayes, G.A. Smith, Biobased Surfactants: Overview and Industrial State of the Art, Second Edi, Elsevier Inc., (2019). https://doi.org/10.1016/b978-0-12-8127056.00001-0.

[3] E.I.P. Delbeke, I.N.A. Van Bogaert, Sophorolipid Modification: The Power of Yeasts and Enzymes Prospects of Practical Application of Sophorolipids, Cellobiose Lipids, and MELs, 18 (2018).

[4] S. Rebello, A.K. Asok, S. Mundayoor, M.S. Jisha, Surfactants: Toxicity, remediation and green surfactants, Environ. Chem. Lett. 12 (2014) 275-287.

https://doi.org/10.1007/s10311-014-0466-2.

[5] J. Vandeputte, Agro-based surfactants, OCL - Ol. Corps Gras Lipides. 19 (2012) 133 137. https://doi.org/10.1051/ocl.2012.0431.

[6] L.Y. Zakharova, T.N. Pashirova, S. Doktorovova, A.R. Fernandes, E. Sanchez-Lopez, A.M. Silva, S.B. Souto, E.B. Souto, Cationic surfactants: Self-assembly, structureactivity correlation and their biological applications, 20 (2019) 1-31. https://doi.org/10.3390/ijms20225534.

[7] K. Akhter, K. Ullah, R. Talat, A. Haider, N. Khalid, F. Ullah, S. Ali, Synthesis and characterization of cationic surfactants and their interactions with drug and metal complexes, Heliyon. 5 (2019) e01885. https://doi.org/10.1016/j.heliyon.2019.e01885.

[8] F. Goursaud, M. Berchel, J. Guilbot, N. Legros, L. Lemiègre, J. Marcilloux, D. Plusquellec, T. Benvegnu, Glycine betaine as a renewable raw material to "greener" new cationic surfactants, Green Chem. 10 (2008) 310-32. https://doi.org/10.1039/b713429k.

[9] Z.F. Nsimba, M. Paquot, L.G. Mvumbi, M. Deleu, Les derivés tensioactifs de la glycine bétaïne: Méthodes de synthèse et potentialités d'utilisation, Biotechnol. Agron. Soc. Environ. 14 (2010) 737-748.

[10] I. Faye, V. Besse, G. David, S. Caillol, Sustainable cardanol-based ionic surfactants, Green Mater. 5 (2017) 144-152. https://doi.org/10.1680/jgrma.17.00018.

[11] M.T. Garcia, O. Kaczerewska, I. Ribosa, B. Brycki, P. Materna, M. Drgas, Biodegradability and aquatic toxicity of quaternary ammonium-based gemini surfactants: Effect of the spacer on their ecological properties, Chemosphere. 154 (2016) 155-160. https://doi.org/10.1016/j.chemosphere.2016.03.109. 
[12] N. Pérez, L. Pérez, M.R. Infante, M.T. García, Biological properties of arginine-based glycerolipidic cationic surfactants, Green Chem. 7 (2005) 540-546.

https://doi.org/10.1039/b419204d.

[13] A. Singh, V.K. Tyagi, Arginine based novel cationic surfactants: A review, Tenside, Surfactants, Deterg. 51 (2014) 202-213. https://doi.org/10.3139/113.110299.

[14] D.B. Tripathy, A. Mishra, J. Clark, T. Farmer, Synthesis, chemistry, physicochemical properties and industrial applications of amino acid surfactants: A review, Comptes Rendus Chim. 21 (2018) 112-130. https://doi.org/10.1016/j.crci.2017.11.005.

[15] I. Aranaz, N. Acosta, C. Civera, B. Elorza, J. Mingo, C. Castro, M. de los L. Gandía, A.H. Caballero, Cosmetics and cosmeceutical applications of chitin, chitosan and their derivatives, Polymers (Basel). 10 (2018) 213. https://doi.org/10.3390/polym10020213.

[16] J. Zhang, W. Xia, P. Liu, Q. Cheng, T. Tahirou, W. Gu, B. Li, Chitosan modification and pharmaceutical/biomedical applications, Mar. Drugs. 8 (2010) 1962-1987. https://doi.org/10.3390/md8071962.

[17] M.S. Rodríguez, L.A. Albertengo, E. Agulló, Emulsification capacity of chitosan, Carbohydr. Polym. 48 (2002) 271-276. https://doi.org/10.1016/S0144-8617(01)002582.

[18] M.A.. P. D.R. Mallawarachchi, A.D.U.S Amarasinghe, Suitability of chitosan as an emusifier for cationic bitumen emulsions and its behavior as an additive to bitumen emulsion, Constr. Build. Mater. 102 (2016) 1-6.

[19] F. Nan, J. Wu, F. Qi, Y. Liu, T. Ngai, G. Ma, Uniform chitosan-coated alginate particles as emulsifiers for preparation of stable Pickering emulsions with stimulus dependence, Colloids Surfaces A Physicochem. Eng. Asp. 456 (2014) 246-252.

https://doi.org/10.1016/j.colsurfa.2014.05.017.

[20] S. Zhang, Y. Zhou, C. Yang, Pickering emulsions stabilized by the complex of polystyrene particles and chitosan, Colloids Surfaces A Physicochem. Eng. Asp. 482 (2015) 338-344. https://doi.org/10.1016/j.colsurfa.2015.06.029.

[21] L. Alison, A. F. Demirörs, E. Tervoort, A. Teleki, J. Vermant, Emulsions Stabilized by Chitosan-Modified Silica Nanoparticles pH, (2018) 6147-6160.

[22] H. Li, Z. Zhang, X. Bao, G. Xu, P. Yao, Fatty acid and quaternary ammonium modified chitosan nanoparticles for insulin delivery, Colloids Surfaces B Biointerfaces. 170 (2018) 136-143. https://doi.org/10.1016/j.colsurfb.2018.05.063.

[23] A. Bani-Jaber, I. Hamdan, M. Alkawareek, The synthesis and characterization of fatty 
acid salts of chitosan as novel matrices for prolonged intragastric drug delivery, Arch. Pharm. Res. 35 (2012) 1159-1168. https://doi.org/10.1007/s12272-012-0706-6.

[24] C. Chapelle, G. David, S. Caillol, C. Negrell, G. Durand, M. Desroches Le Foll, S. Trombotto, Water-Soluble 2,5-Anhydro-D-mannofuranose Chain End Chitosan Oligomers of a Very Low Molecular Weight: Synthesis and Characterization, Biomacromolecules. 20 (2019) 4353-4360. https://doi.org/10.1021/acs.biomac.9b01003.

[25] Z. Song, Y. Wen, P. Deng, F. Teng, F. Zhou, H. Xu, S. Feng, L. Zhu, R. Feng, Linolenic acid-modified methoxy poly (ethylene glycol)-oligochitosan conjugate micelles for encapsulation of amphotericin B, Carbohydr. Polym. 205 (2019) 571-580. https://doi.org/10.1016/j.carbpol.2018.10.086.

[26] Y.Z. Du, L. Wang, H. Yuan, F.Q. Hu, Linoleic acid-grafted chitosan oligosaccharide micelles for intracellular drug delivery and reverse drug resistance of tumor cells, Int. J. Biol. Macromol. 48 (2011) 215-222. https://doi.org/10.1016/j.ijbiomac.2010.11.005.

[27] C. Chapelle, G. David, S. Caillol, C. Negrell, G. Durand, M.D. le Foll, Functionalization of Chitosan Oligomers: From Aliphatic Epoxide to Cardanol-Grafted Oligomers for Oilin-Water Emulsions, Biomacromolecules. 22 (2021) 846-854. https://doi.org/10.1021/acs.biomac.0c01576.

[28] K. Takamura, A. James, Paving with asphalt emulsions, Advances in Asphalt Materials, (2015) 393-422. https://doi.org/10.1016/B978-0-08-100269-8.00013-1.

[29] D. Day, I.M. Lancaster, D. McKay, Emulsion cold mix asphalt in the UK: A decade of site and laboratory experience, J. Traffic Transp. Eng. (English Ed.) 6 (2019) 359-365. https://doi.org/10.1016/j.jtte.2019.05.002.

[30] R.A. Sheldon, Metrics of Green Chemistry and Sustainability: Past, Present, and Future, ACS Sustainable Chemistry and Engineering, 6 (2018) 32-48. https://doi.org/10.1021/acssuschemeng.7b03505.

[31] O. Mengual, G. Meunier, I. Cayre, K. Puech, P. Snabre, Characterisation of instability of concentrated dispersions by a new optical analyser: The TURBISCAN MA 1000, Colloids Surfaces A Physicochem. Eng. Asp. 152 (1999) 111-123. https://doi.org/10.1016/S0927-7757(98)00680-3.

[32] M. J. Rosen, Surfactants and Interfacial Phenomena, (1989) 548. https://doi.org/10.1080/01932699008943278.

[33] P. Mukerjee, The nature of the association aquilibria and hydrophobic bonding in aqueous solutions of association colloids, Adv. Colloid. Interface Sci. 1 (1967) 241- 
275.

[34] C. Della Volpe, S. Siboni, The Wilhelmy method: A critical and practical review, Surf. Innov. 6 (2018) 120-132. https://doi.org/10.1680/jsuin.17.00059.

[35] D.J. Burgess, N.O. Sahin, Interfacial rheological and tension properties of protein films, J. Colloid Interface Sci. 189 (1997) 74-82.

https://doi.org/10.1006/jcis.1997.4803.

[36] Y. Wei, Y. Xie, Z. Cai, Y. Guo, M. Wu, P. Wang, R. Li, H. Zhang, Interfacial and emulsion characterisation of chemically modified polysaccharides through a multiscale approach, J. Colloid Interface Sci. 580 (2020) 480-492. https://doi.org/10.1016/j.jcis.2020.07.048.

[37] H. Yuan, L.J. Lu, Y.Z. Du, F.Q. Hu, Stearic acid-g-chitosan polymeric micelle for oral drug delivery: In vitro transport and in vivo absorption, Mol. Pharm. 8 (2011) 225-238. https://doi.org/10.1021/mp100289v.

[38] A. Almeida, M. Araújo, R. Novoa-Carballal, F. Andrade, H. Gonçalves, R.L. Reis, M. Lúcio, S. Schwartz, B. Sarmento, Novel amphiphilic chitosan micelles as carriers for hydrophobic anticancer drugs, Mater. Sci. Eng. C. 112 (2020) 110920. https://doi.org/10.1016/j.msec.2020.110920.

[39] N. Querol, C. Barreneche, L.F. Cabeza, Storage stability of bimodal emulsions vs. monomodal emulsions, Appl. Sci. 7 (2017). https://doi.org/10.3390/app7121267.

[40] X. Gutierrez, F. Silva, M. Chirinos, J. Leiva, H. Rivas, Bitumen-in-water emulsions: An overview on formation, stability, and rheological properties, J. Dispers. Sci. Technol. 23 (2002) 405-418. https://doi.org/10.1080/01932690208984213.

[41] Pérez, Lourdes, Pinazo, Aurora, Teresa García, M., Lozano, Marina, Manresa, Angeles, Angelet, Marta, Pilar Vinardell, M., Mitjans, Montse, Pons, Ramon, Rosa Infante, M. European Journal of Medicinal Chemistry 44 (2009) 1884-1892 